\title{
Effects of Orchidectomy on Bone Metabolism in Beagle Dogs
}

\author{
Satoshi FUKUDA ${ }^{1,2)}$ and Haruzo IIDA ${ }^{2)}$ \\ 1) International Space Radiation Laboratory and ${ }^{2)}$ Division of Radiotoxicology and Protection, National Institute of Radiological \\ Sciences, 4-9-1 Anagawa, Inage-ku, Chiba 263-8555, Japan
}

(Received 17 March 1999/Accepted 17 September 1999)

\begin{abstract}
The effects of orchidectomy on bone metabolism in male beagle dogs were examined using twelve 2-year-old dogs that were orchidectomized. The dogs' bilateral iliac bones, double-labeled with tetracycline and calcein for the histomorphometry, were obtained from three dogs prior to orchidectomy and at 3, 6, 9, and 12 months afterwards. The serum biochemical constituents related to bone metabolism were examined before and every month after orchidectomy. Between 1 and 6 months after orchidectomy, the value of serum testosterone decreased (1 month), while the levels of parathyroid hormone, calcitonin, total calcium, osteocalcin, and alkaline phosphatase activity increased significantly, indicating a high bone turnover. The mean trabecular thickness and the fraction of labeled osteoid surface decreased significantly 3 months after orchidectomy, but other histomorphometric parameters were unchanged. In the period 7-12 months after orchidectomy, the parathyroid hormone level increased ever and above that of the first 6-month period, while the levels of calcitonin, osteocalcin, alkaline phosphatase activity, and phosphorus decreased. The bone volume, mean trabecular thickness, and the fraction of labeled trabecular surface decreased significantly compared with the pre-orchidectomy values. These findings indicate an imbalance in bone metabolism ( i.e. bone resorption > bone formation). These results indicate that a loss of bone volume accompanied the fall in sex hormone levels following orchidectomy and suggest that the orchidectomized dog is available as an animal model for studying osteoporosis caused by hypogonadism and the decline of sex functions in men.-KEY wORDs: bone histomorphometry, canine, iliac bone, orchidectomy.
\end{abstract}

It has been noted in many studies that osteoporosis occurs in postmenopausal women due to the reduction or loss of sex hormones $[8,10,15]$. While osteoporosis in men might not be so frequent because sex hormones and functions decrease slowly with aging in males, however, it does occur in men with hypogonadism $[2,11]$. This indicates that the loss or reduced levels of sex hormones, such as androgen and testosterone, causes osteoporosis in men. To our knowledge, there are few reports on osteoporosis in men and in male dogs. The mechanisms by which these hormones influence bone metabolism have not yet been elucidated [11]. In order to investigate osteoporosis caused by the loss or fall in male hormones, the establishment of a suitable animal model is necessary.

Colony-raised beagle dogs are a useful experimental animal for this purpose as the characteristics of their bone metabolic pattern are similar to those in humans [3], because the bone metabolism pattern at the tissue level in both dogs and humans changes from the modeling pattern in the growth process to the remodeling pattern after they reach maturity or adulthood [3]. Also, the bone growth plate in beagle dogs disappears at about 14 months, indicating that bone growth ceases after dogs reach maturity [7]. All histomorphometric values, e.g., bone volume and bone formation rate, showed adult states after at least 12 months old [5]. We also reported in a previous study that serum testosterone levels in male beagle dogs remained high with a regular circadian rhythm from 6 months to 12 years, and that the levels increased further from 13 to 16 years [6]. This indicates that osteoporosis due to the alterations of sex hormones and functions, such as those that occur with age, do not occur spontaneously in male dogs. We therefore used orchidectomized dogs in this study in order to investigate the effects of orchidectomy on bone metabolism in male beagle dogs. We examined these effects by carrying out a histomorphometric study, using undecalcified sections of the iliac trabecular bone and by measuring serum biochemical constituents related to bone metabolism.

\section{MATERIALS AND METHODS}

Animals: Twelve male beagle dogs aged 2 years, an age when the bone metabolism is in an adult states, were obtained from the beagle breeding colony established under the genetic and quality controls in our institute. After about 6-8 months of age, the animals were housed in stainless steel cages. Up to that age they were allowed to exercise freely in larger cages. Almost no individual variations in bone histomorphometric values or in serum constituents related to bone metabolism which usually show considerable variation due to environmental and nutrient factors, were found in the dogs bred in our colony [4]. The animals were given water ad libitum, and $250 \mathrm{~g}$ of laboratory dog food daily (DM-1, Funabashi Farm Co., Ltd.), which contained calcium (2.0\%), phosphorus (1.6\%), and vitamin D (200 UI in $100 \mathrm{~g}$ ). The bilateral testicles were removed from all dogs under anesthesia with a combination of ketamine hydrochloride $25 \mathrm{mg} / \mathrm{kg}$ (Sankyo Co., Ltd.) and xylazine 10 $\mathrm{mg} / \mathrm{kg}$ (Bayer Co., Ltd.).

Bone labeling and histomorphometry: Before orchidectomy, all dogs were injected intramuscularly twice with tetracycline hydrochloride $(25 \mathrm{mg} / \mathrm{kg}$, Pfizer Co., Ltd.) at 7-day intervals for double labeling the bone surface calcification so as to obtain dynamic parameters for the 
histomorphometry. Seven days after the second tetracycline injection, prior to the orchidectomy, the left iliac bone was extracted by biopsy with a trephine ( $8 \mathrm{~mm}$ internal diameter) at a site $1.5 \mathrm{~cm}$ below and behind the anterior superior iliac spine. At 3, 6, 9, and 12 months after orchidectomy, three dogs were injected subcutaneously with calcein $(8 \mathrm{mg} / \mathrm{kg}$, Nakarai Chemicals Ltd.) at 7-day intervals. Seven days after the second calcein injection, the right iliac bone was extracted at the site symmetrical to the left extracted site before orchidectomy.

The undecalcified bone specimens were immersed in Villanueva's bone stain solution [20], dehydrated in ethanol and acetone, and embedded in methylmethacrylate. The block was subsequently cut into thin sections with an innerblade cutter (MC-808D, Maruto Instrument Co., Ltd.). The sample was ground to a thickness of about $15 \mu \mathrm{m}$ by grinding glass plates (Asahi Glass Co., Ltd.). The histomorphometric measurements of the cancellous bone area of the ilium were carried out with a semi-automatic image analyzer using the software "Osteoplan II" (Kontron 64, Carl Zeiss Co.) under fluorescent microscopy [13]. The parameters measured and the units of measurement were as follows: bone volume/tissue volume (BV/TV, \%), trabecular thickness (Tb.Th, $\mu \mathrm{m}$ ), osteoid volume/tissue volume (OV/ $\mathrm{TV}, \%)$, osteoid thickness (O.Th, $\mu \mathrm{m})$, double labeled surface/osteoid surface (dLS/OS, \%), double labeled surface/ bone surface (dLS/BS, \%), mineral apposition rate (MAR, $\mu \mathrm{m} /$ day), bone formation rate/bone volume (BFR/BV, \%/ year) and mineralization lag time (Mlt, day) [16].

Measurement of serum biochemical constituents: Blood was obtained from the left brachiocephalic vein before and every month after orchidectomy. The blood sample was clotted and centrifuged for $15 \mathrm{~min}$ at 3,000 r.p.m. The serum sample was separated and frozen at $-20^{\circ} \mathrm{C}$ and then stored for later analysis. The serum biochemical constituents that were measured and the methods of measurement used were: total calcium concentration (o-cresolphthalein complexone method, calcium C-test, Wako Pure Chemical Co., Ltd.) and phosphorus concentration (molybdenum blue method, phosphor B-test, Wako Pure Chemical Co., Ltd.), which were determined with a spectrophotometer (Model 200-20; Hitachi Ltd.); alkaline phosphatase activity (Bessey-Lowry's modified method; Yamanouchi-Boehringer Mannheim), which was determined with an autoanalyzer (AKES reaction rate analyzer; Vitaron Co., The Netherlands); and serum immunoreactive parathyroid hormone concentration (PTH), which was determined by radioimmunoassay using a chicken anti-C-terminal human parathyroid hormone (Yamasa Co.). The calcitonin concentration was also determined by radioimmunoassay (Daiichi Isotope Co.), as was the bone $\gamma$ carboxyglutamic acid containing protein (BGP, also called osteocalcin) concentration (radioimmunoassay developed by Asahi Chemical Co., Ltd.). These parameters were checked, incompletely, for cross reactivities between dogs and humans, and with each other. Finally the testosterone concentration was also determined by radioimmunoassay (Eiken Co.) [6].
Statistical analysis: The statistical analyses to determine the significance of the differences between values before and after the orchidectomy were the Mann-Whitney U test and the Wilcoxon test.

Ethics: The experimental animals used in this study were treated and/or handled according to the "Recommendations for the Handling of Laboratory Animals for Biomedical Research", compiled by the Committee on the Safety and Ethical Handling Regulations for Laboratory Animal Experiments at our institute.

\section{RESULTS}

Serum testosterone levels decreased rapidly 1 month after orchidectomy and was less than the detectable limit $(0.5 \mathrm{ng} /$ $\mathrm{ml}$ ) 2 months after the operation (Fig. 1). There were significant increases in body weight at 4,11 and 12 months compared to the values before orchidectomy (Fig. 2). PTH increased rapidly up to 4 months and tended to increase thereafter (Fig. 3). Calcitonin had increased at 3 months, but decreased thereafter and returned to the base line at 912 months (Fig. 3). ALP increased during the first 7 months and then decreased to a level less than base line during 911 months (Fig. 4). BGP activity increased during the first 6 months and maintained a high level till 12 months, although it decreased at 7 months and then increased again till 12 months (Fig. 4). Total calcium increased and reached a peak at 6 months, then decreased but maintained a high and constant level (Fig. 5). Phosphorus did not change significantly during the first 6 months but was lower after 7 months than the value before orchidectomy (Fig. 5).

The mean values in the bone histomorphometry are shown in Table 1. There was a significant decrease in bone volume at 12 months compared to the value before orchidectomy (Fig. 6). There were also significant decreases in the mean trabecular thickness at 3,9 and 12 months compared to the value before orchidectomy. A significant difference was observed in the osteoid thickness at 3 months after orchidectomy as compared to before it. There was a significant decrease in the fraction of the double-labeled trabecular surface at 9 months compared to the values of before orchidectomy.

\section{DISCUSSION}

Although the precise mechanisms responsible for the action of sex steroids on bone metabolism are still unknown [11], it has been shown that male sex hormones such as androgen, dihydrotestosterone, and testosterone stimulate mineralization and increase mineral content in bone and bone volume $[9,17]$, whereas $17 \beta$-estradiol suppresses bone formation in male dogs in puberty [19]. Arisaka et al. [2, 11] suggest that androgens are responsible for preventing bone resorption in adults. In fact, a lower bone mass accompanied by a decrease in serum testosterone level is observed in males with hypogonadism. Male sex hormone treatment does affect mineralization. Kapur and Reddi [9] 


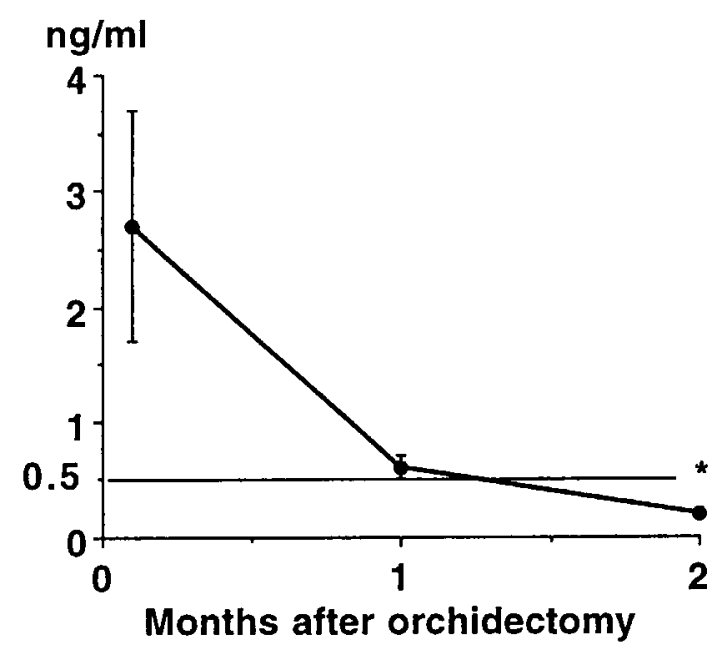

Fig. 1. Fall in serum testosterone level after orchidectomy. The mean value before the orchidectomy $(2.7 \pm 1.0 \mathrm{ng} / \mathrm{ml})$ declined to $0.6 \pm 0.1 \mathrm{ng} / \mathrm{m} l$ in nine dogs post-orchidectomy. Less than the $0.5 \mathrm{ng} / \mathrm{m} l$ detectable limit $(*)$ was present in three dogs 1 month post-orchidectomy; less than $0.5 \mathrm{ng} / \mathrm{m} l$ was present in nine dogs and $0.5-0.8 \mathrm{ng} / \mathrm{m} l$ was present in three dogs 2 months post-orchidectomy. Vertical bars indicate standard deviation.

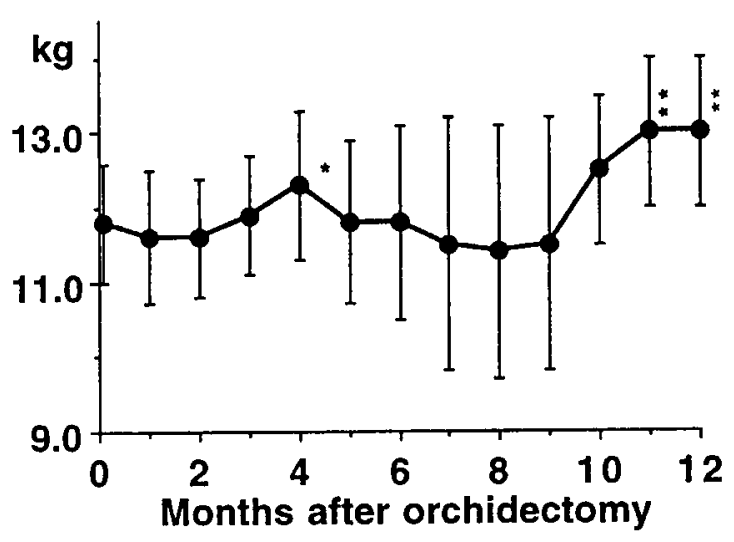

Fig. 2. Changes in body weight after orchidectomy. The mean body weight increased significantly at $4(\mathrm{p}<0.05)$, 11 and 12 months $(\mathrm{p}<0.01)$ compared to the values before orchidectomy. Vertical bars indicate standard deviation.

indicated that androgen and dihydrotestosterone stimulate mineralization for short periods of time and in the early stage of bone development in spayed rats. The various effects of metabolites of male sex hormones on bone metabolism may appear over time as changes in the serum biochemical parameters and bone histomorphometric values in this study. However, slight changes in bone histomorphometric values during the early period after orchidectomy were not detectable within the limits of the present study, although they may actually begin to vary in

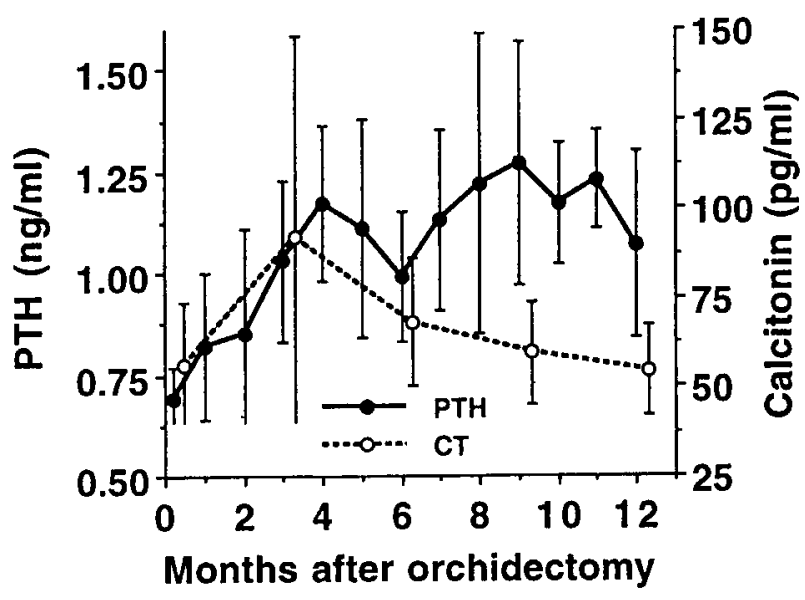

Fig. 3. Changes in serum PTH and calcitonin concentrations after orchidectomy. PTH increased significantly at 1 month $(\mathrm{p}<0.05)$ and between 3 and 12 months $(\mathrm{p}<0.001)$ compared to the value before the orchidectomy. Calcitonin was elevated significantly at 3 months $(\mathrm{p}<0.05)$, however it had returned to the baseline level 9 months later. Vertical bars indicate standard deviation.

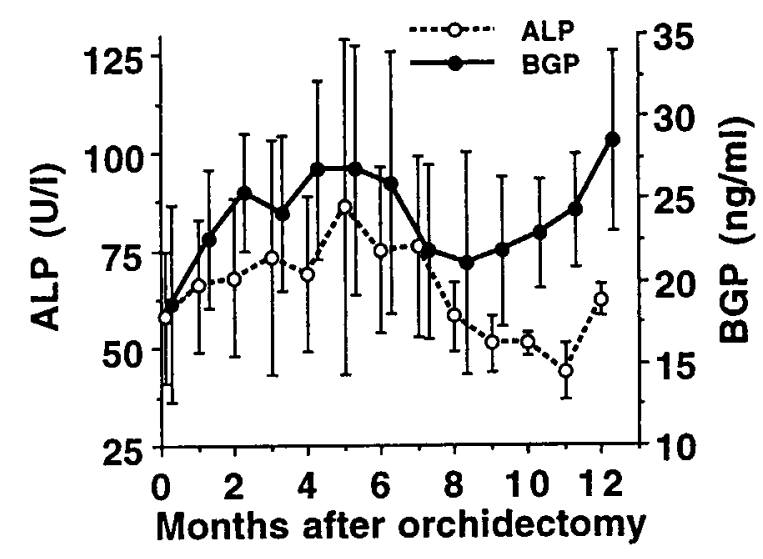

Fig. 4. Changes in serum alkaline phosphatase (ALP) activity and BGP after orchidectomy. ALP increased during the first 7 months and then decreased to a value less than base line during 9-11 months. BGP increased at 2 months $(\mathrm{p}<0.01), 3$ months $(\mathrm{p}<0.05), 4$ months $(\mathrm{p}<0.01)$, and 5 and 6 months $(\mathrm{p}<0.05)$, and then decreased after 7 months, and increased again till 12 months. Vertical bars indicate standard deviation.

response to the changes in serum biochemical parameters.

The processes responsible for the bone volume loss that occurred in the orchidectomized dogs in this study can be divided into the following two stages, based on the changes in serum biochemical constitutes and bone volume (Figs. 36). In the first stage (the first 6 months after orchidectomy), increased PTH and calcitonin levels might have been caused by the loss of male sexual hormones induced by orchidectomy; first PTH was elevated, and this was followed by the elevation of calcitonin which then suppressed the action of PTH. Simultaneously, high levels of PTH and 


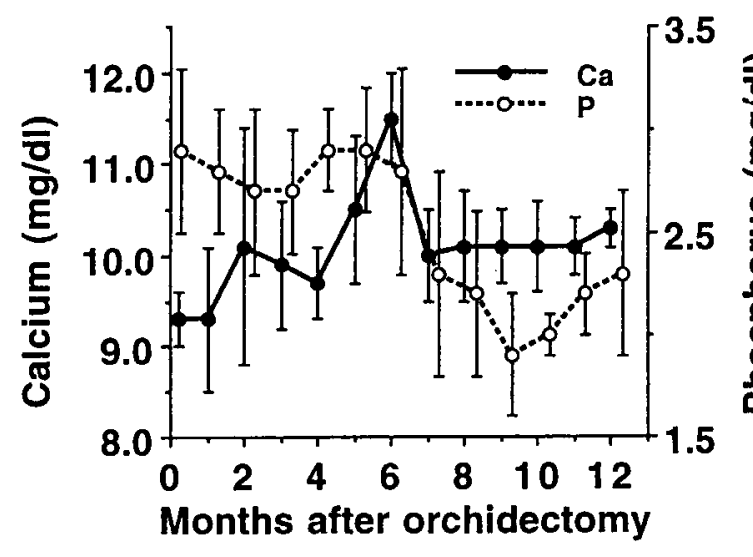

Fig. 5. Changes in serum total calcium and phosphorus after orchidectomy. Total calcium increased significantly at 3 and 4 months $(\mathrm{p}<0.05), 5$ and 6 months $(\mathrm{p}<0.001), 7$ and 8 months $(p<0.01), 9$ months $(p<0.001), 10$ months $(p<0.01)$, and 11 and 12 months $(\mathrm{p}<0.001)$. Phosphorus decreased significantly at 7 and 8 months $(\mathrm{p}<0.01), 9$ months $(\mathrm{p}<0.001), 10$ and 11 months $(\mathrm{p}<0.01)$ and 12 months $(\mathrm{p}<0.05)$ compared to the value before orchidectomy. Vertical bars indicate standard deviation.

calcitonin appear to compete to maintain serum calcium level. The increases in BGP and alkaline phosphatase activity indicate that osteoblasts may have been activated. However, the significant increase of serum total calcium might be due to bone resorption generated by the action of PTH being greater than bone formation generated by the action of calcitonin, i. e. the higher bone turnover. In short, these changes in serum biochemical parameters in the first stage indicate an imbalance of bone resorption and formation, that resulted in a decrease of the mean trabecular thickness and in the fraction of labeled osteoid seams, although other histomorphometric parameters were not significantly changed. Namely, it may be that the histological changes observed at 3 months were still not advanced to a state which could be detected later by the

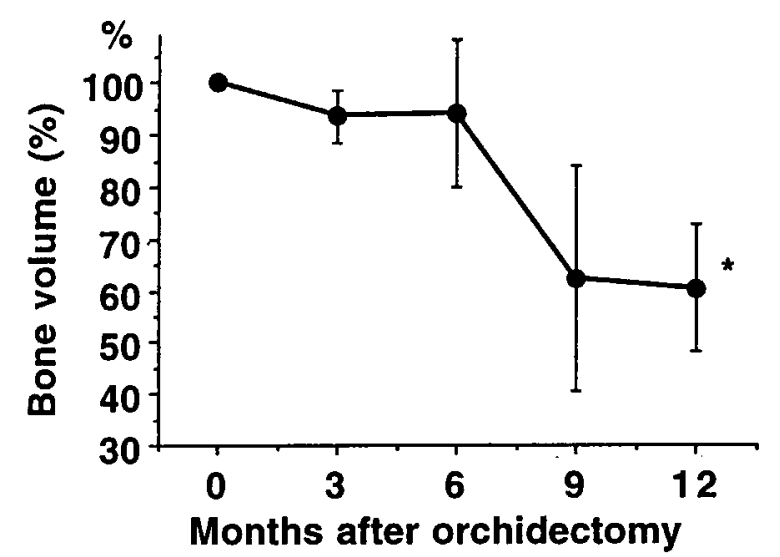

Fig. 6. Decrease in bone volume (BV/TV) after orchidectomy, shown as percentages of the values before orchidectomy in each group. There was a significant decrease in bone volume $(\mathrm{p}<0.05)$ at 12 months compared to the value of before orchidectomy.

histomorphometric method. These obscure results may also be related to the unchanged intestinal absorption of calcium by the dietary sufficient calcium supplementation in the orchidectomized dogs, because testosterone is not related to vitamin D and its metabolites [12].

During the second stage (the 7-12 months after orchidectomy), the PTH level was more elevated than in the first stage, while the levels of calcitonin, alkaline phosphatase activity, BGP, and phosphorus decreased. High levels of PTH indicate bone resorption, while decreases in calcitonin, alkaline phosphatase activity and BGP indicate the decline of osteoblast activity. Also, dysfunction of osteoblast activity may be induced by the loss of estrogen because dysfunction of osteoblast activity is known to occur in beagle dogs after high bone turnover induced by ovariectomy $[14,18]$. These changes in serum constituents indicate that bone resorption distinctly exceeded bone formation. These unusual changes in the various serum

Table 1. Histomorphometric values obtained before and after the orchidectomy

\begin{tabular}{|c|c|c|c|c|c|c|c|c|c|}
\hline Time & $\begin{array}{c}\mathrm{BV} / \mathrm{TV} \\
(\%)\end{array}$ & $\begin{array}{l}\text { Tb.Th } \\
(\mu \mathrm{m})\end{array}$ & $\begin{array}{c}\mathrm{OV} / \mathrm{TV} \\
(\%)\end{array}$ & $\begin{array}{l}\text { O.Th } \\
(\mu \mathrm{m})\end{array}$ & $\begin{array}{c}\mathrm{dLS} / \mathrm{OS} \\
(\%)\end{array}$ & $\begin{array}{c}\mathrm{dLS} / \mathrm{BV} \\
(\%)\end{array}$ & $\begin{array}{c}\text { MAR } \\
(\mu \mathrm{m} / \text { day })\end{array}$ & $\begin{array}{l}\text { BFR/TV } \\
\text { (\%/year) }\end{array}$ & $\begin{array}{c}\text { Mlt } \\
\text { (days) }\end{array}$ \\
\hline Before ${ }^{\#}$ & $26.9 \pm 1.4$ & $331 \pm 23$ & $4.78 \pm 2.66$ & $11.15 \pm 1.25$ & $0.748 \pm 0.054$ & $0.070 \pm 0.036$ & $1.01 \pm 0.21$ & $41.8 \pm 24.0$ & $11.5 \pm 3.1$ \\
\hline 3 months & $25.2 \pm 2.5$ & $282 \pm 16^{*}$ & $5.27 \pm 1.68$ & $7.45 \pm 1.2^{*}$ & $1.037 \pm 0.573$ & $0.137 \pm 0.056$ & $0.91 \pm 0.02$ & $82.6 \pm 27.2$ & $8.2 \pm 1.4$ \\
\hline Before $^{\#}$ & $27.5 \pm 7.1$ & $306 \pm 44$ & $4.80 \pm 1.03$ & $9.91 \pm 2.77$ & $0.956 \pm 0.135$ & $0.097 \pm 0.020$ & $0.96 \pm 0.13$ & $55.5 \pm 4.8$ & $10.3 \pm 2.5$ \\
\hline 6 months & $25.2 \pm 2.8$ & $260 \pm 7$ & $4.05 \pm 2.49$ & $5.70 \pm 1.31$ & $0.543 \pm 0.240$ & $0.057 \pm 0.021$ & $0.87 \pm 0.29$ & $36.2 \pm 1.5$ & $6.9 \pm 1.6$ \\
\hline Before ${ }^{\#}$ & $31.2 \pm 2.9$ & $374 \pm 50$ & $4.11 \pm 2.98$ & $8.71 \pm 3.73$ & $2.002 \pm 0.949$ & $0.153 \pm 0.021$ & $1.06 \pm 0.11$ & $81.6 \pm 25.3$ & $8.2 \pm 3.5$ \\
\hline 9 months & $19.8 \pm 8.7$ & $268 \pm 36^{*}$ & $6.45 \pm 5.17$ & $9.38 \pm 0.90$ & $0.630 \pm 0.313$ & $0.078 \pm 0.03 *$ & $0.92 \pm 0.15$ & $51.8 \pm 22.8$ & $10.0 \pm 2.4$ \\
\hline Before ${ }^{\#}$ & $33.1 \pm 7.1$ & $359 \pm 17$ & $7.17 \pm 3.67$ & $11.48 \pm 2.06$ & $1.184 \pm 1.118$ & $0.122 \pm 0.093$ & $0.93 \pm 0.38$ & $49.7 \pm 37.4$ & $13.4 \pm 4.1$ \\
\hline 12 months & $19.6 \pm 3.1 *$ & $288 \pm 27^{*}$ & $5.47 \pm 2.14$ & $9.69 \pm 2.20$ & $0.773 \pm 0.204$ & $0.101 \pm 0.008$ & $0.89 \pm 0.10$ & $58.6 \pm 10.9$ & $10.8 \pm 1.6$ \\
\hline
\end{tabular}

Values are mean \pm standard deviation.

* Significant difference in values after the orchidectomy compared to the pre-values $(\mathrm{P}<0.05)$.

\# Values before orchidectomy in each observed group. 
biochemical constituents resulted in acute reductions of bone volume and trabecular thickness and in a decline in bone formation or mineralization. These were indicated by significant reductions in the double-labeled trabecular surface fraction and decreases (although not significant) in mineral apposition and bone formation rates. Although the dogs' body weight increased significantly at 11 and 12 months after orchidectomy, these increases cannot be attributed to prevention of bone loss. This is because the rate of increase was within 10 percent of the preorchidectomy rate and because all the dogs used in this study were kept in cages and had restricted exercise during the experimental period. Indeed, Wronski et al. [21] have shown that body weight did not influence the decrease in bone volume in ovariectomized rats.

Although our findings have demonstrated that the loss of sex hormones produced by orchidectomy in male dogs successfully changed both the serum biochemical and histomorphometric parameters, which suggests the occurrence of osteoporosis, the histomorphometric and serum biochemical parameters did not always change in ovariectomized dogs $[14,18]$. Such sex differences may be due to the maintenance of high levels of sex hormones such as testosterone in male dogs [6], while in female dogs, the intervals in the estrus cycle are long (220 \pm 56 days) and the increases in sex hormones during estrus occur for a short time (only 7-14 days) [1]. Alternatively, there may be differences in the effects of male and female sex hormones on bone metabolism.

The results obtained in this study demonstrate that osteoporosis occurs in orchidectomized beagle dogs, as shown by the changes in bone histomorphometric values and serum biochemical parameters. These findings indicate that orchidectomized adult dogs can be utilized as a model of osteoporosis in which the interactions of male sex hormones and bone metabolism, including bone remodeling, can be investigated. Treatment for osteoporosis that accompanies either hypogonadism or the reductions in or loss of sex hormones and function in men can therefore be examined in this model. Also the preventions (e.g. by dietary calcium supplement and exercise) should be studied in orchidectomized dogs.

\section{REFERENCES}

1. Andersen, A. C. 1970. Reproduction. pp. 31-39. In: The Beagle as an Experimental Dog (Andersen, A. C, ed.), The Iowa State Univ. Press, Iowa.

2. Arisaka, M., Arisaka, O., Hosaka, A., Tokita, A., Shimura, N., Yabuta, K. and Kawaguchi, Y. 1989. Osteoporosis in a boy hydrogonadotropic hypogonadism. JBMM. 7: 18-22.

3. Frost, H. M. 1973. Remodeling as a determinant of envelope physiology. pp. 28-53. In: Bone Remodeling and Its Relationship to Metabolic Bone Disease (Frost, H. M. ed.), Charles C Thomas, Springfield.

4. Fukuda, S. and Iida, H. 1991. Comparison of histomorphometric values in iliac trabecular bone of beagle dogs raised under different breeding system. Exp. Anim. (Tokyo) 41: 131-137.

5. Fukuda, S. and Iida, H. 1994. Changes in histomorphometric values of iliac trabecular bone and serum biochemical constituents related to bone metabolism in beagle dogs during growth. Exp. Anim. (Tokyo) 43: 159-165.

6. Fukuda, S., Nagashima, H., Morioka, K. and Aoki, J. 1988. Fluctuations in peripheral serum testosterone levels within a day, with age and by sexual stimulation in male beagle dogs bred indoors. Exp. Anim. (Tokyo) 37: 381-386.

7. Ichiki, H. 1976. pp. 119-174. Radiographic studies on normal skeleton growth. In: Studies on Beagle for Research in Japan (Fukui,M., Tomoda, I. and Ueda, K. eds), Soft Science, Tokyo.

8. Kanis, J. A. 1996. pp. 71-195. Assessment of bone mass. In: Textbook of Osteoporosis. Blackwell Sciences, London.

9. Kapur, S. P. and Reddi, A. H. 1989. Influence of testosterone and dihydrotestosterone on bone-matrix induced endochondral bone formation. Calcif Tissue Int. 44: 108-113.

10. Kassem, M., Melton III, L. J. and Giggs, B. L. 1996. pp. 691-701. The type I/typeII model for involutional osteoporosis. In: Osteoporosis (Marcus, R., Feldman, D. and Kelesey, J. eds.), Academic press, San diego.

11. Kitajima, I., Amitani, H., Fukunaga, H., Iwata, S., Kubota, R., Izumo, S. and Osame, S. 1989. Two cases of male hypogonadal osteoporosis. JBMM. 7: 42-47.

12. Krabbe, S., Hummer, L. and Christiansen, C. 1986. Serum levels of vitamin D metabolites and testosterone in male puberty. JCE \& M. 62: 503-507.

13. Malluche, H. H., Sherman, D., Meyer, W. and Massary, S. G. 1982. A new semiautomatic method for quantitative static and dynamic bone histology. Calcif Tissue Int. 34: 439-448.

14. Martin, R. B., Butcher, R. L., Sherwood, L. L., Buckendahl, P., Boyd, R. D., Farris, D., Sharkey, N. and Dannucci, G. 1987. Effects of ovariectomy in beagle dogs. Bone 8: 23-31.

15. Meunier, P. J. 1995. Bone histomorphometry. pp 299-318. In: Osteoporosis: Etiology, Diagnosis, and Management (Riggs, B. L. and Melton III, L. J. eds.), Lippincott-Reven, Philadelphia.

16. Parfitt, A. M., Drezner, M. K., Glorieux, F. H., Kanis, J. A., Malluche, H., Meunier, P. J., Ott, S. M. and Recker, R. R. 1987. Bone histomorphometry: Standardization of nomenclature, symbols, and units. J. Bone Mineral Res. 2: 595-609.

17. Riis, B. J., Krabbe, S., Christiansen, C., Catherwood, B. D. and Deftos, L. J. 1985. Bone turnover in male puberty: A longitudinal study. Calcif Tissue Int. 37: 213-217.

18. Shen, V., Dempster, D. W., Birchman, R., Mellish, R. W. E, Church, E., Kohn, D. and Lindsay, R. 1991. Lack of changes in histomorphometric, bone mass, and biochemical parameters in ovariohysterectomized dogs. Bone 13: 311-316.

19. Snow, G.R. and Anderson, C. 1985. The effects of continuous estradiol therapy on cortical bone remodeling activity in the spayed beagle. Calcif Tissue Int. 37: 282-286.

20. Villanueva, A. R. 1974. A bone stain for osteoid seams in fresh, unembedded mineralized bone. Stain Technol. 49: 1-8.

21. Wronski, T. J., Schenck, P. A., Cintrón, M. and Walsh, C. C. 1987. Effects of body weight on osteopenia in ovariectomized rats. Calcif Tissue Int. 40: 155-159. 